\title{
Psychophysical effects in double-slit interference patterns: Response to a critique
}

\author{
Dean Radin ${ }^{1,2,}$ Helané Wahbeh ${ }^{2,3}$, Leena $_{\text {Michel}}^{2}$, and Arnaud Delorme ${ }^{2,4}$ \\ 1 California Institute of Integral Studies, San Francisco, CA \\ 2 Institute of Noetic Sciences, Petaluma, CA \\ 3 Oregon Health \& Science University, Portland, OR \\ 4 University of California, San Diego
}

Version: August 3, 2020

Correspondence concerning this article should be addressed to

Dean Radin, Institute of Noetic Sciences, Petaluma, CA 94954 dean@noetic.org 


\section{ABSTRACT}

An experiment was conducted to explore possible psychophysical influences on interference patterns generated by an optical double-slit system. The experiment was motivated by a proposed explanation of the quantum measurement problem by John von Neumann and others, namely that consciousness collapses the quantum wavefunction.

The study design generated a single database partitioned into eight differential tests, four in experimental conditions where participants alternately directed their attention toward and away from the optical system, and four in sham conditions when no one was present. The planned analysis was comparison of means in attention-toward vs. attention-away conditions; a secondary analysis added later, but before the data were examined, was comparison of variances. After adjusting the eight mean comparisons for multiple testing, none were statistically significant, but one of the eight variance differences was suggestively significant.

To date, 11 of 28 conceptual replications of this experiment, reported by four independent teams, have reported statistically significant outcomes $(p<0.05$, two-tail), where only one would be expected by chance. Given the implications of psychophysical effects on our understanding of the role of consciousness in the physical world, we encourage others to attempt to replicate this experiment.

Keywords: consciousness, psychophysical, mind-matter interactions, double-slit 


\section{INTRODUCTION}

The concept that matter influences mind is accepted as the orthodox assumption underlying the neurosciences and psychopharmacology (Wessinger \& Clapham, 2009). Likewise, the possibility that mind influences matter within the body is also acceptable and studied within the disciplines of psychophysiology and psychoneuroimmunology (Nes \& Segerstrom, 2017). By contrast, the idea that the mind also influences matter outside the body arouses a wide range of opinions, ranging from enthusiasm to outrage (Alcock et al., 2003). However, it is not a new idea.

Mind as primary over matter is a core concept in both the Western and Eastern esoteric traditions (Huxley, 1945). It is even found within the writings of Francis Bacon, widely considered to be the founder of modern empiricism (Glebkin, 2019). For example, in Sylva Sylvarum, Bacon discussed methods of studying the "force of imagination," his term for direct mind-matter interactions (Bacon \& Rawley, 1639).

In more recent times, questions about Bacon's "force of imagination" have reappeared in a new form. As physicist Richard Feynman famously said, it resides in "the heart of quantum mechanics" (Feynman et al., 1971). Feynman was referring to the fact that photons behave differently when they are observed versus not observed. This phenomenon is at the core of the "quantum measurement problem," which violates the common-sense notion that physical reality does not depend on being observed. That is, most people believe that the moon is still there even when you are not looking at it.

The quantum measurement problem was discussed by nearly all the founders of quantum mechanics. They thought deeply about its epistemological and ontological challenges, and some, like Planck, Pauli, Eddington, Jordan, Jeans, Gödel, London, Wigner, and von Neumann, proposed that consciousness may be responsible in some way for the transition from quantum possibilities into classical actualities (Rosenblum \& Kuttner, 2006). As Jordan wrote, "Observations not only disturb what is to be measured, they produce it. We compel [the electron] to assume a definite position. We ourselves produce the results of measurement" (cited in Mermin, 1990). 
Discussions about the relationship between physics and consciousness, like many enduring topics in science and scholarship, have passed through periods ranging from fallow to intense interest. In recent decades, there may be signs of a renaissance (Atmanspacher et al., 2015; Stapp, 2011). Changes in opinions are also reflected in the writings of contemporary physicists who insist that mind-matter interaction phenomena are impossible, but at the same time they find themselves asking in a New York Times op-ed, "Is consciousness somehow involved in the basic rules of reality?" (Carroll, 2019; emphasis in the original). This idea may also be gaining new respectability because of recent (mainstream) experimental evidence that supports the idea that quantum theory "should be interpreted in an observer-dependent way" (Proietti et al., 2019, p. 1).

One of the more prominent interpretations of the quantum measurement problem was proposed by John von Neumann, who described the measurement process as a chain of quantum interactions between physical entities. This quantum chain could only be "cut" and become classical actualities when knowledge of the measurement was registered by what von Neumann called an "extra-physical" or "psychophysical" factor, i.e., the observer's mind (von Neumann, 1955). To test this idea, some have proposed to use the human eye as a type of quantum detector (Brunner et al., 2008). But the eye, and for that matter the brain, are physical entities, not extraphysical, so those types of detectors would not cut the measurement chain. To take von Neumann's proposal seriously, it is necessary to use the "mind's eye," or subjective imagination, as the observer.

\section{Previously reported experiments}

The first experiment using a double-slit optical system to test von Neumann's proposal was reported by Jeffers (1996). A few years later, Ibison independently replicated the experiment using a refined version of Jeffers' apparatus, and then they jointly reported their results in this journal (Ibison \& Jeffers, 1998). In those experiments, participants were invited to use "extrasensory perception" to mentally gain information about the path of a photon inside a double-slit system. If that effort were successful, then it would presumably cause a change in the double-slit interference pattern. Jeffers reported a null effect; Ibison reported a successful outcome ( $p<0.05$, one-tailed). 
Starting in 2009, we began a series of studies designed to replicate and extend the Jeffers/Ibison experiments. Our studies used both custom-made and commercially available double-slit optical systems (Radin et al., 2012; Radin et al., 2013; Radin et al., 2015; Radin et al, 2016). Another series of replications was recently reported by Guerrer (2019). In this article, we discuss a similar experiment we were funded to conduct in 2012 by Jan Walleczek, the scientific director of a private foundation.

Some readers may wonder why we waited 8 years (as of this writing) to report this study. Shortly after the experiment was finished, we prepared a report describing the results, but Walleczek requested that we not publish it at that time. Given that WS have since published an article on the study, we felt it was now appropriate to also publish our account. In particular, many procedural details of our experiment were not described in WS, so for the sake of completeness and to help facilitate possible future replications, we present that information here.

In a separate publication, we will report the results of a post-hoc analysis requested by Walleczek after we had completed the initial analysis. To accommodate his request required the development of a new metric, which fortunately turned out to be much simpler than the original approach, and it also provided a clearer interpretation of the results.

\section{METHOD}

\section{Hypotheses}

The primary hypothesis was that observation, in the form of mental attention directed toward a double-slit interference pattern, would result in a "collapse" of the quantum wavefunction. The proposed effect would be detected as a decrease in the double-slit spectral component of the interference pattern, as compared to the same measure when attention was withdrawn. This directional hypothesis assumes that focused attention can be turned on and off like a switch, and it is stable and consistent among different participants. However, many people find that maintaining a single point of focused attention for more than a few minutes is challenging (Smallwood \& Schooler, 2006), so to assist in focusing on the task in this experiment, participants tended to adopt more active strategies, such as trying to mentally push photons through one of the two slits, or trying to mentally block photons from passing through one of the slits. This means the task entailed variations in both attention and intention. 
Previous research on intentional mind-matter interaction tasks indicates that intentional tasks can sometimes result in outcomes opposite to the assigned instructions, depending on unconscious factors and demand characteristics of the experiment (Debes \& Morris, 1982; Stanford, 1981; Varvoglis \& Bancel, 2015). These reversals suggest that a directional hypothesis may not be fully realized, thus a more general secondary hypothesis was considered (importantly, before examining the data), namely that the effect would manifest as a nondirectional change in the interference pattern, as measured by a shift in variance.

\section{Equipment}

The experiment took place inside the Institute of Noetic Sciences' double steel-walled electromagnetically shielded chamber (Series 81 Solid Cell, ETS-Lindgren Cedar Park, TX, USA). Besides the custom-made optical apparatus, thermocouples continuously monitored ambient temperature (with $0.1^{\circ} \mathrm{F}$ accuracy) on the laser tube, on the housing of the optical apparatus, between the participant and the apparatus, and at the back of the chair where the participant sat, about 2 meters from the apparatus.

\section{Metric}

Each interference pattern image recorded by the camera was transformed by a Fast Fourier Transform (Matlab function $f f t$ ) to determine the image's power spectrum, and then the real portion of the spectrum, its "power" $\boldsymbol{p}$, was $\log 10$-transformed to reduce skew. Images of the interference pattern and associated power spectrum may be seen in Radin et al $(2012,2013)$. In the optical system used for this test, the spectral power associated with the double-slit component of the interference pattern peaked at wavenumber 45; call this $\boldsymbol{p}_{\mathbf{4 5}}$. Examination of $\boldsymbol{p}_{\mathbf{4 5}}$ showed that the laser's illumination intensity randomly hopped between two power regimes (so-called "mode-hopping" is common in HeNe gas lasers).

To reduce the variance associated with mode-hopping, which produces a bimodal distribution of $\boldsymbol{p}_{\mathbf{4 5}}$, the analysis employed an algorithm described in Radin et al (2013). It involved first detrending the power spectrum between peaks associated with single-slit diffraction and double-slit interference, specifically between the nearly linear portion of the spectrum between wavenumbers 14 and 39. Then the resulting values at wavenumber 45 were linearly detrended across all spectral measures recorded per session. This "double-detrended" 
power variable $\left(\boldsymbol{p}_{d \boldsymbol{d}}\right)$ was the metric used in subsequent analyses. The approach was useful in that it adequately normalized the data, but adjustment of the power spectrum also made the results difficult to interpret in physical terms. As mentioned above, a post-hoc metric (described in a separate publication) requested by Walleczek substantially simplified interpretation of the outcome.

\section{Protocol}

Call $\mathbf{X}$ the instruction to focus attention toward the double-slit system, and $\mathbf{O}$ the instruction to withdraw attention. Each attention epoch lasted 30 seconds, and these two types of instructions were used to create 4 epoch-pair conditions: OO, XX, XO and OX. A single test session consisted of 20 epoch-pairs in the form of 5 repetitions of these 4 conditions, with each pair presented in a newly randomized sequential order in each session. Within an epoch-pair, each epoch was separated from the next by a few seconds, and each epoch-pair was separated from adjacent pairs by a randomized latency of 3 to 5 seconds. The latter was incorporated to decouple successive epoch-pairs from potential cyclic behavior in laser power.

Under the primary hypothesis, comparison of the mean of $\boldsymbol{p}_{d \boldsymbol{d}}$ samples within an $\mathbf{O O}$ epoch-pair was not expected to reveal a difference because neither the first nor the second $\mathbf{O}$ in the epoch-pair involved mental observation. For $\mathbf{X X}$ pairs, a null mean difference was also expected, but only under the assumption that the observer effect was stable and uniformly applied across in each epoch. Comparisons of means in $\mathbf{O X}$ and $\mathbf{X O}$ pairs were expected to result in positive and negative differences, respectively (i.e., the mean in the first epoch compared to the mean in the second epoch).

Because epoch-pairs were presented in random orders, it was possible that as part of a session a participant could be presented with the sequence of instructions: ... OX XX XO... That sequence would require maintaining focused concentration for two minutes, with just a few short breaks. Given the difficulty in maintaining one-pointed attention for that length of time, we were not optimistic that a directional hypothesis would be supported. But we did anticipate that differences in epoch-pair variance might still be detectable. This variance analysis was not planned during the initial study design, which is perhaps why it is not mentioned in WS's critique. But it was also not a post-hoc prediction because it was added prior to decryption and examination of the data. 
Besides conducting an experimental test of the four epoch-pair conditions, a second, identically designed "sham" experiment was run without observers present. This provided a way to test the effects of the environment, optical apparatus and analytical procedures for possible systematic errors. Data from the sham tests were not expected to show either mean or variance differences for any of the four epoch-pair conditions.

This general design, which to our knowledge has not been used in previous mind-matter interaction studies, was proposed by Walleczek. It is similar to methods commonly used in biological experiments to help distinguish between the causal effects of, say, a toxin on a cell culture, versus systematic errors that might mimic those effects (Lipsitch et al., 2010).

\section{Analytical Plan}

To avoid assumptions about the distribution or possible temporal dependencies among $\boldsymbol{p}_{d \boldsymbol{d}}$ samples, the means of $\boldsymbol{p}_{d \boldsymbol{d}}$ in each epoch-pair were compared using a nonparametric randomized permutation technique (Hoffman, 2019). As part of this analytic procedure, the data were lagged by 3 seconds, a latency selected based on previous lab-based experiments. The purpose of the lag was to account for the cognitive processing time it takes to switch attention from one task to another (Schneider \& Logan, 2009). The outcomes of the permutation technique were expressed in terms of standard normal deviates (i.e., $z$ scores).

Each test session consisted of 5 repetitions of each epoch-pair condition, thus to provide a single summary $z$ score for each condition, the $5 z$ scores per condition were combined into a single Stouffer Z (Stouffer et al, 1949). The result per session was thus eight $z$ scores, one each for the OO, XX, OX, and XO conditions in experimental sessions and the same four comparisons for sham sessions. The secondary, variance hypothesis, was tested by squaring these $z$ scores to form chi-squared distributed values.

\section{Procedure}

An hour before each participant was scheduled to arrive at the lab, a research assistant (RA) switched on the laser power and computer and prepared the Matlab program used to control the experiment. After the warmup period, the RA closed the shielded room and started the first sham session, which used a freshly randomized sequence of epoch-pair conditions. During those sessions a lamp with a 60-watt bulb was placed in the chair where the participants 
would later sit during the experimental sessions. That wattage was selected to generate about the same amount of heat as the participants' bodies. Headphones, which would be used by the participants, were also placed on the chair to keep the environment of the experimental and sham tests as similar as possible.

The first time a participant arrived at the lab, the RA explained the nature of the task, which required them to focus their attention or intention toward the slits inside the optically sealed double-slit system. It was explained that they could try to mentally "see" or imagine which of the two slits the photons were passing through, to "become one with" the optical system in a contemplative way, to mentally block one of the two slits, or to mentally "bend" the laser beam so as to cause it to pass through one slit rather than both. The preferred strategy was selected by the participant, and they were allowed to switch strategies at will. Note that two of the more commonly used strategies involved passive attention, and two involved active intention. Based on discussions with participants in previous studies (Radin et al, 2012), it appeared that most people found it easier to use intentional rather than purely attentional strategies. Once seated, participants were not allowed to move from their chair, and they were instructed to remain quiet and still during the experiment.

While the first sham test was running, participants waited outside the shielded chamber where they were asked to complete the PANAS mood-reporting questionnaire (Positive And Negative Affect Scale, Crawford \& Henry, 2004). When the sham session finished, the RA escorted them to the chair inside the chamber, they put on noise-cancelling headphones, and they began their first experimental session. That session used the same epoch-pair sequence that was randomly generated for the preceding sham test.

Previously recorded voice prompts, played by the computer that controlled the experiment, provided on-going instructions to the participant during the session. Experimental sessions began with the spoken phrase, "The experiment is about to begin, please relax." Data collection then began for 30 seconds to ensure that the equipment was working properly, and if it was then the beginning of each subsequent epoch-pair was announced by the phrase, "a new trial is starting." If the epoch-pair was $\mathbf{O O}$, then the instruction before the first 30-second epoch was "now you may relax," and before the second 30-second epoch it was "please continue to relax." If the epoch pair was $\mathbf{X X}$, the associated phrases were, "now please concentrate," and "please 
continue to concentrate." For an $\mathbf{O X}$ pair, the instructions were "now please relax" before the first epoch, and "now please concentrate" before the second epoch. For an XO pair, the same instructions were presented in reverse order.

At the end of each epoch, the computer spoke, "end of this trial," followed by a short pause and then "please wait for the next trial." Then there was a 10-second break, and when the next pair was about to begin, the phrase was "a new trial is starting." At the end of the entire session, the phrase was "the experiment will be over soon, please remain seated."

After the first experimental session, participants took a break outside the shielded chamber for about 10 minutes. Then they reentered the chamber and completed a second experimental session using a newly randomized sequence of epoch-pair instructions. When the second experimental session was finished, the RA escorted them outside the chamber and started a second sham session using the same epoch-pair sequence used in the preceding experimental session. At this point, the participant completed a post-session PANAS scale. Each session took about 25 minutes, so participants spent at least an hour for each of the five times they visited the lab to complete their series of 10 sessions (see Figure 1).

\begin{tabular}{|l|c|c|c|l|l|l|}
\hline PANAS pre & Sham 1 & Experiment 1 & Break & Experiment 2 & Sham 2 & PANAS post \\
\hline
\end{tabular}

Figure 1. Laboratory Protocol. PANAS = Positive and Negative Affect Scale.

In most of our other double-slit experiments, participants were provided with real-time performance feedback during the focused attention epochs in the form of visual displays or audio tones. The feedback signal was typically associated with the magnitude of the double-slit component of the Fourier spectrum, which was in turn calculated by the computer in real-time. In this study, we did not provide real-time feedback because after we demonstrated the experiment to the first participant, he complained that the feedback was distracting. We occasionally received similar comments from other participants in these experiments, so in an attempt to optimize conditions to allow for unbroken concentration, we turned the real-time feedback off in this study. Instead, at the end of each session the computer informed the participant whether their overall results were, or were not, in alignment with the hypothesis that the grand mean of data during $\mathbf{X}$ epochs would be lower than the same measure during $\mathbf{O}$ epochs 
(because during the $\mathbf{X}$ epochs the double-slit component of the interference spectrum would hypothetically "collapse," or decline).

\section{Participants}

The experimental design specified that 25 participants would each contribute 10 experimental and 10 matched sham sessions, for a total of 500 sessions. Individuals recruited for the test were selected based either on their performance on previous experiments of this type, or because they maintained an active meditation practice or other form of mental discipline that require focused attention, such as musician, healer, or artist. The study was approved by the IONS Institutional Review Board and all participants signed an informed consent before beginning the experiment. Participants were informed that upon successfully completing all 10 sessions, they would be compensated for their time and efforts with a $\$ 100$ check.

\section{Procedural integrity}

The randomly generated epoch-pair assignments were encrypted and stored in real-time. This ensured that the data could not be analyzed until all planned sessions were completed, at which time the decryption password would be provided by Walleczek. As planned, the encryption code was finally broken in March 2013 after all data were collected. The data analysis was performed in the presence of Walleczek. Copies of the raw data and the decrypted epochpair assignments were then handed to Walleczek ( 80 GBytes).

Because interferometers are sensitive to environmental influences, participants listened to the attention assignments over noise-cancelling headphones to reduce ambient vibrations and potential electromagnetic interference. The headphones also ensured that the RA, who was located outside the chamber, could not accidentally overhear those assignments and possibly influence the participants by say, fidgeting more during $\mathbf{X}$ epochs versus $\mathbf{O}$ epochs. In addition, to help maintain systematic interactions with participants across all sessions, the same RA ran all experimental and sham sessions.

\section{RESULTS}

As planned, 25 participants successfully contributed 10 sessions each, for a total of 250 experimental and 250 sham sessions. The first 20 people participated from February 2012 
through September 2012, and then due to unforeseen circumstances the lab was relocated from a quiet ground floor location to a noisier second-floor location. That move introduced a threemonth delay, so the last 5 participants were run from January 2013 through February 2013. The new environment was not as comfortable as the original site, as reflected in a significant increase in the PANAS negative affect score from a mean (and standard error) of $11.3 \pm 0.17$ for the first 200 sessions to $13.2 \pm 0.54$ for the last 50 sessions ( $p=0.001$ two-tailed).

As mentioned above, each session produced eight $z$ scores, so $250 \times 8=2,000 z$ scores were generated in the experiment. Inspection of those scores, using both Lilliefors and DurbinWatson statistics (Matlab R2019a functions lillietest and dwtest), showed that the $z$ scores were normally distributed and independent.

The results are shown in Table 1. When the $p$-values for the eight mean comparison tests are adjusted for multiple testing, none achieve significance at $p<0.05$ after applying the False Discovery Rate (FDR) method (Benjamini \& Hochberg, 1995). Thus, this experiment provided no evidence for a directional psychophysical effect. When the $p$-values for the eight variance comparison tests were adjusted with FDR, one outcome was significant at $p<0.05$ in the experimental OX condition, which was one of the two conditions predicted to show a difference. Based on these results, it appeared that this study provided partial evidence in favor of the second hypothesis. We note that Guerrer (2019), in his series of formal, pre-registered double-slit experiments, also observed a significant non-directional effect, post-hoc. If one argued that FDR should be applied to $16 \mathrm{p}$-values rather than 8 , then none of the comparisons would have achieved significance, so the variance outcome is best regarded as suggestive. 
Table 1. Results of the planned analysis at lag -3 seconds, as shown in the Matlab analysis script provided on request by the funders of the experiment (Walleczek \& von Stillfried, 2019).

\begin{tabular}{|l|l|l|l|l|l|l|l|l|}
\hline & \multicolumn{3}{|l|}{ Experiment } & \multicolumn{1}{l|}{ Sham } & & \\
\hline condition & $\mathrm{OO}$ & $\mathrm{XX}$ & $\mathrm{OX}$ & $\mathrm{XO}$ & $\mathrm{OO}$ & $\mathrm{XX}$ & $\mathrm{OX}$ & $\mathrm{XO}$ \\
\hline $\begin{array}{l}z \text { (mean } \\
\text { comparisons) }\end{array}$ & -0.75 & 0.72 & -1.28 & -0.66 & -1.62 & 1.82 & 1.14 & -1.92 \\
\hline$p$ (2-tail) & 0.45 & 0.47 & 0.20 & 0.51 & 0.11 & 0.07 & 0.25 & 0.05 \\
\hline $\begin{array}{l}\text { Chi Square } \\
\text { (variance } \\
\text { comparisons) }\end{array}$ & 268.9 & 270.7 & 260.6 & 309.5 & 252.7 & 278.3 & 250.3 & 251.6 \\
\hline$p$ (chi) & 0.20 & 0.18 & 0.31 & 0.006 & 0.44 & 0.11 & 0.48 & 0.46 \\
\hline
\end{tabular}

$\mathbf{X}$ - Concentrate epochs; $\mathbf{O}$ - Relax epochs

Prompted by the intriguing result in the variance analysis, we explored whether the $\mathrm{z}$ scores in the OX condition correlated with those in the $\mathrm{XO}$ condition. The reasoning was that if focused attention produced genuine mean-shifts within sessions, but they were not uniformly in the same direction across sessions, then the correlation between OX vs. XO values should be negative. That is, within a session the OX condition should shift the mean in one direction and the XO condition in the other direction. The same correlation evaluated in sham data should be null.

As predicted, the OX vs XO correlation for the experimental data was significantly negative, $\mathrm{r}_{\mathrm{E}}=-0.14, \mathrm{p}=0.03$ (two-tail), while the same correlation for the sham data was nonsignificant, $\mathrm{r}_{\mathrm{C}}=-0.02, \mathrm{p}=0.71$. This finding triggered a more general analysis to examine all 28 unique pairwise correlations among the 8 conditions in the experimental and sham data. Of the resulting correlations, 4 were independently significant, and all of them were in the experimental data. After application of FDR, the 3 correlations that remained significant were $r(X X$ vs $O X)=$ $-0.20, p=0.002, r(X X$ vs. OX $)=-0.19, p=0.003$, and $r(X X$ vs $X O)=-0.18, p=0.004$.

We then investigated whether the attentional stability assumed by the primary hypothesis was supported by the PANAS mood data. We were particularly interested in the PANAS "positive mood" and "attentiveness" subscales. Both measures showed that participants' mood 
significantly declined over sessions, with the former correlation $r=-0.28, d f=248, p=6 \times 10^{-6}$, and the latter correlation $r=-0.22, d f=248, p=0.0005$. Given that the task in this experiment required focused attention, we speculate that the experimental protocol, environment, or both, were suboptimal. Previous studies of this type that we conducted typically required participants to run one or two sessions to avoid burnout. Thus, in hindsight, requiring 10 repeated sessions was probably a shortcoming of this study design.

\section{DISCUSSION}

As mentioned above, WS published a critique of this experiment. Among other concerns, they claimed that a false-positive result in a sham condition "casts doubt on the scientific validity of the claimed (true)-positive effect which has been reported before (Radin et al., 2012, 2013, 2015, 2016)." (WS, p. 17). That assertion is problematic for two reasons: First, it ignores the statistical inflation caused by conducting eight comparisons. As we noted above, when the eight $p$-values were adjusted for multiple comparisons, none were significant, thus WS's claim about a false-positive is itself false. To be clear, this is not a matter of mere opinion. WS's failure to adjust for multiple comparisons is a mathematical mistake, confirmed by a former president of the American Statistical Association (Utts, October 2019, personal communication). As we have previously explained,

"If each of eight comparisons used $\mathrm{p}<0.05$ as the conventional threshold for rejecting the null hypothesis, then the probability of obtaining at least one false-positive is $p=1-$ $(0.95)^{8}=0.34$, or $34 \%$. In other words, one or more false-positives would be identified one third of the time, even in data that were pure noise." (Radin et al., 2020, p. 1).

Second, WS's critique blithely dismissed 28 similar studies reported to date, including experiments conducted by three other independent groups (Guerrer, 2019; Jeffers, 1996; Ibison \& Jeffers, 1998). Eleven of those 28 studies reported significant outcomes ( $<<0.05$, two-tailed), where just one would be expected to be significant by chance. This is extremely unlikely to have occurred by chance $\left(\mathrm{p}<10^{-7}\right)$.

WS's claim is further unwarranted because these studies: (1) involved different optical apparatus, sources of illumination, cameras, and methods of analysis; (2) included both exploratory and planned formal protocols; (3) investigated variables predicted to correlate with 
human performance, including participants' beliefs, EEG alpha power, and global geomagnetic flux (Radin et al., 2012, 2015); (4) found predicted lags in the results due to the cognitive costs associated with shifting attention toward and away from the optical system; (5) found no deviations from chance in control tests designed to test the apparatus, protocols, and analyses for systematic biases; and (6) reported all data to avoid selective reporting biases.

WS claimed that: "Radin and co-workers have routinely interpreted $z$-scores on the order of two sigma as representing significant evidence for an anomalous consciousness effect" ( $p$. 15). This assertion is notably ironic because it was not us, but rather WS who relied on a single unadjusted two-sigma (i.e., $\mathrm{p}$ < 0.05) effect for the basis of their critique. In fact, of the 16 double-slit experiments we conducted, five produced results greater than four sigma (Radin et al., 2012, experiment 5; Radin et al., 2013, experiments 1 and 2; Radin et al., 2015, experiment 1; and Radin et al., 2016, experiment 1).

WS then recommended that researchers "discontinue the exploratory research approach toward the Radin DS-experiment and ... start implementing the methods and protocols that are consistent with strictly confirmatory research practices and designs." (p. 17). We disagree because given the results observed in several of our experiments, as well as positive evidence observed in independent experiments, a more fruitful use of limited resources would be to continue exploratory studies in addition to pre-registered replications. It might make sense to pursue confirmatory studies if this were a mature line of experimentation with a well-tested protocol. But as we have emphasized in our publications, the hypothesis under study is a purported psychophysical effect. Given that it is not possible to "step in the same river twice" when studying ordinary psychological phenomena, if one is serious about probing the enigmatic interstitial space between psychology and physics, then we believe it is woefully premature to narrow one's focus to only include confirmatory studies.

Finally, WS suggested that: "Skepticism should replace optimism concerning the radical claim that an anomalous quantum consciousness effect has been observed in a controlled laboratory setting." We might agree with that sentiment if the present line of research were the only relevant evidence. However, this is not the case. The experiments discussed here are part of a research program on mind-matter interactions that began some nine decades ago (Rhine, 1944). A recent meta-analysis of over 1,000 relevant published experiments showed that each of five 
classes of psychophysical interaction studies resulted in small magnitude but statistically significant, repeatable outcomes (Cardeña, 2018). In any case, skepticism and optimism are not antonyms. Skepticism is a core feature of scientific epistemology, but equally important are curiosity, exploration, and enthusiasm. We can think of few things more depressing than striving to maintain a state of doubtful pessimism while exploring a fascinating topic at the edge of the known.

\section{CONCLUSION}

Critical debates over methods and findings are essential in sharpening scientific work, and as such we grateful for WS's critiques, as well as those by other authors who have commented on our studies (Baer, 2015; Pallikari, 2015; Pradhan, 2015; Sassoli de Bianchi, 2013; Tremblay, 2019). We hope that presentation of our findings will encourage others to attempt their own replications. If independent studies can confirm the presence of genuine mind-matter interactions, especially within the context of informing interpretations of the quantum measurement problem, then the implications for understanding the role of consciousness in the physical world would be profound. In attempting these replications, it is worth bearing in mind Francis Bacon's sage advice about jumping to conclusions one way or the other when exploring the "force of imagination":

Men are to [be] admonished that they do not withdraw credit from operations by ... force of imaginations, because the effects fail sometimes.... The relations touching the force of imagination, and the secret instincts of nature, are so uncertain, as they require a great deal of examination ere we conclude upon them (cited in Montagu, 1841, p. 124).

\section{ACKNOWLEDGEMENTS}

For their support of one or more of our double-slit experiments, we gratefully acknowledge the Fundação Bial, the Federico and Elvia Faggin Foundation, Inc., the Hittman Family Foundation, the Emerald Gate Charitable Trust, the John B. Huntington Foundation, Klee Irwin and Quantum Gravity Research, Richard and Connie Adams, and the donors and members of the Institute of Noetic Sciences. 


\section{REFERENCES}

Alcock, J. E., Burns, J., \& Freeman, A. (Eds.). (2003). Psi Wars: Getting to Grips with the Paranormal. Imprint Academic.

Atmanspacher, H., Biernacki, L., Carr, B., Fach, W., Grosso, M., Murphy, M. F., Presti, D. E., Shaw, G. L., Stapp, H. P., \& Weiss, E. M. (2015). Beyond Physicalism: Toward Reconciliation of Science and Spirituality. Rowman \& Littlefield.

Bacon, F. (1639). Sylva Sylvarum or a Naturall Historie. William Rawley.

Baer, W. (2015). Independent verification of psychophysical interactions with a double-slit interference pattern. Physics Essays, 28, 47-54.

Benjamini, Y., \& Hochberg, Y. (1995). Controlling the False Discovery Rate: A Practical and Powerful Approach to Multiple Testing. Journal of the Royal Statistical Society. Series B (Methodological), 57(1), 289-300. https://doi.org/10.2307/2346101

Brunner, N., Branciard, C., \& Gisin, N. (2008). Possible entanglement detection with the naked eye. Physical Review A, 78(5), 052110. https://doi.org/10.1103/PhysRevA.78.052110

Cardeña, E. (2018). The experimental evidence for parapsychological phenomena: A review. American Psychologist, 73(5), 663-677. https://doi.org/10.1037/amp0000236

Carroll, S. (2019, September 7). Opinion | Even Physicists Don't Understand Quantum Mechanics. The New York Times. https://www.nytimes.com/2019/09/07/opinion/sunday/quantum-physics.html

Crawford, J. R., \& Henry, J. D. (2004). The positive and negative affect schedule (PANAS): Construct validity, measurement properties and normative data in a large non-clinical sample. The British Journal of Clinical Psychology, 43(Pt 3), 245-265. https://doi.org/10.1348/0144665031752934

Dean Radin, Leena Michel, Alan Pierce, \& Arnaud Delorme. (2015). Psychophysical interactions with a single-photon double-slit optical system. Quantum Biosystems, 6(1), 82-98.

Dean Radin, Leena Michel, Karla Galdamez, Paul Wendland, Robert Rickenbach, \& Arnaud Delorme. (2012). Consciousness and the double-slit interference pattern: Six experiments. Physics Essays, 25(2).

Debes, J., \& Morris, R. (1982). Comparison of striving and nonstriving instructional sets in a PK study. Journal of Parapsychology, 46(4), 297-312. 
Feynman, R. P., Leighton, R. B., \& Sands, M. (1971). The Feynman Lectures on Physics, Vol. 3 (Later Printing edition). Addison Wesley.

Glebkin, V. (2019). Cultural-historical underpinnings of early modern science: The case of Francis Bacon and René Descartes. International Journal of Critical Cultural Studies, 17(1), 89-102. Scopus. https://doi.org/10.18848/2327-0055/CGP/v17i01/89-102

Guerrer, G. (2019). Consciousness-related interactions in a double-slit optical system. https://doi.org/DOI 10.17605/OSF.IO/QDKVX

Hoffman, J. I. E. (2019). Chapter 37-Resampling Statistics. In J. I. E. Hoffman (Ed.), Basic Biostatistics for Medical and Biomedical Practitioners (Second Edition) (pp. 631-637). Academic Press. https://doi.org/10.1016/B978-0-12-817084-7.00037-1

Huxley, A. (1945). The Perennial Philosophy. Harper Colophon Books.

Ibison, M., \& Jeffers, S. (1998). A double-slit diffraction experiment to investigate claims of consciousness-related anomalies. Journal of Scientific Exploration, 12, 543.

Jeffers, S. (1996). Intentionality and complementarity: What might the double slit experiment tell us about consciousness? Toward a Science of Consciousness II, Tucson, Arizona.

Lipsitch, M., Tchetgen Tchetgen, E., \& Cohen, T. (2010). Negative controls: A tool for detecting confounding and bias in observational studies. Epidemiology, 21(3), 383-388. https://doi.org/10.1097/EDE.0b013e3181d61eeb

Mermin, N. (1990). Boojums All the Way through: Communicating Science in a Prosaic Age (1 edition). Cambridge University Press.

Montagu, B. (1841). The works of Francis Bacon, Lord Chancellor of England. Vol. II. Carey and Hart.

Nes, L. S., \& Segerstrom, S. C. (2017). Psychoneuroimmunology 光. In Reference Module in Neuroscience and Biobehavioral Psychology. Elsevier. https://doi.org/10.1016/B978-012-809324-5.22186-X

Pallikari, F. (2015). On the question of wavefunction collapse in a double-slit diffraction experiment. ArXiv:1210.0432 [Quant-Ph]. http://arxiv.org/abs/1210.0432

Pradhan, R. K. (2015). An explanation of psychophysical interactions in the quantum double-slit experiment-Physics Essays Publication. Physics Essays, 28(3), 324-330. 
Proietti, M., Pickston, A., Graffitti, F., Barrow, P., Kundys, D., Branciard, C., Ringbauer, M., \& Fedrizzi, A. (2019). Experimental test of local observer independence. Science Advances, 5(9), eaaw9832. https://doi.org/10.1126/sciadv.aaw9832

Radin, D. I., Michel, L., Galdamez, K., Wendland, P., Rickenbach, R., \& Delorme, A. (2012). Consciousness and the double-slit interference pattern: Six experiments. Physics Essays, 25(2).

Radin, D., Johnston, J., \& Delorme, A. (2013). Psychophysical interactions with a double-slit interference pattern. Physics Essays, 26(4), 553-566.

Radin, D., Michel, L., \& Delorme, A. (2016). Psychophysical modulation of fringe visibility in a distant double-slit optical system. Physics Essays, 29(1), 14-22.

Radin, D., Michel, L., Pierce, A., \& Delorme, A. (2015). Psychophysical interactions with a single-photon double-slit optical system. Quantum Biosystems, 6(1), 82-98.

Radin, Dean, Wahbeh, H., Michel, L., \& Delorme, A. (2020). Commentary: False-Positive Effect in the Radin Double-Slit Experiment on Observer Consciousness as Determined With the Advanced Meta-Experimental Protocol. Frontiers in Psychology, 11. https://doi.org/10.3389/fpsyg.2020.00726

Rosenblum, B., \& Kuttner, F. (2006). Quantum enigma: Physics encounters consciousness. Oxford University Press.

Sassoli de Bianchi, M. (2013). Quantum measurements are physical processes. Comment on "Consciousness and the double-slit interference pattern: Six experiments," by Dean Radin et al. [Phys. Essays 25, 157 (2012)]. Physics Essays, 26(1), 15-20.

Schneider, D. W., \& Logan, G. D. (2009). Task Switching. In L. R. Squire (Ed.), Encyclopedia of Neuroscience (pp. 869-874). Academic Press. https://doi.org/10.1016/B978008045046-9.00426-5

Smallwood, J., \& Schooler, J. W. (2006). The restless mind. Psychological Bulletin, 132(6), 946-958.

Stanford, R. G. (1981). "Associative activation of the unconscious" and "visualization" as methods for influencing the PK target: A second study. Journal of the American Society for Psychical Research, 75(3), 229-240.

Stapp, H. P. (2011). Mindful Universe: Quantum Mechanics and the Participating Observer (2nd ed.). Springer-Verlag. https://www.springer.com/gp/book/9783642180750 
Stouffer, S. A., Suchman, E. A., DeVinney, L. C., Star, S. A., \& Williams, R. M. (1949). The American soldier: Adjustment during army life (Vol. 1). Princeton University Press.

Tremblay, N. (2019). Independent re-analysis of alleged mind-matter interaction in double-slit experimental data. PLOS ONE, 14(2), e0211511. https://doi.org/10.1371/journal.pone.0211511

Varvoglis, M., \& Bancel, P. A. (2015). Micro-psychokinesis. In Parapsychology: A handbook for the 21 st century (pp. 266-281). McFarland \& Co.

von Neumann, J. (1955). Mathematical Foundations of Quantum Mechanics. Princeton University Press.

Walleczek, J., \& von Stillfried, N. (2019). False-Positive Effect in the Radin Double-Slit Experiment on Observer Consciousness as Determined With the Advanced MetaExperimental Protocol. Frontiers in Psychology, 10. https://doi.org/10.3389/fpsyg.2019.01891

Wessinger, C. M., \& Clapham, E. (2009). Cognitive Neuroscience: An Overview. In L. R. Squire (Ed.), Encyclopedia of Neuroscience (pp. 1117-1122). Academic Press. https://doi.org/10.1016/B978-008045046-9.00299-0 